\title{
Hermitian-to-quasi-Hermitian quantum phase transitions
}

\author{
Miloslav Znojil \\ Nuclear Physics Institute CAS, 25068 Řě̌, Czech Republic \\ znojil@ujf.cas.cz \\ http://gemma.ujf.cas.cz/ znojil/
}

\begin{abstract}
The phenomenon of quantum phase transition is considered in the special case in which the evolution laws remain unitary and in which the bound-state energies remain observable. The conventional Hermiticity of observables is lost at the interface, replaced by the so called quasi-Hermiticity. Several features of the passage of the system through the interface are discussed and illustrated by elementary illustrative $\mathcal{P} \mathcal{T}$-symmetric examples.
\end{abstract}

\section{Keywords}

unitary quantum theory; non-Hermitian representation; evolution equations; phase transitions; PT symmetric benchmark model; 


\section{Introduction}

In historical retrospective the birth of quantum mechanics was certainly facilitated by several remarkably friendly experimental as well as theoretical aspects of its applicability, say, to hydrogen atom or to elementary molecules [1]. Even the transition from nonrelativistic hydrogen-atom-like Hamiltonians $\mathfrak{h}$ to their relativistic Dirac-equation amendments $\widetilde{\mathfrak{h}}$ remained smooth, straightforward and compatible with the parallel refinements of the measurements of the stationary bound-state energy spectra. An immanent limitation of applicability of the traditional self-adjoint local-interaction models emerged only after a next-step transition to the description of the motion of a relativistic electron (or electrons) in some perceivably stronger (e.g., heavy-ion) central Coulomb potential $\widetilde{\mathfrak{v}}=-Z e^{2} /|\vec{r}|$. At the overcritical effective charges with $Z>1 / \alpha \approx 137$, due to the so called Klein paradox, the system crossed the boundary of stability and entered a "mathematically forbidden" zone. The ground-state energy ceased to be observable since it acquired a non-vanishing imaginary part (cf., e.g., pp. 195 - 206 in [2] for details). Similar problems also occurred in the case of Klein-Gordon equation (cf. [3]).

The loss-of-observability process of the degeneracy and of the subsequent complexification of the energy levels can be interpreted as a non-conservative quantum phase transition [4, 5]. In the language of physics the phenomenon is traditionally attributed to the emergence of a new relevant degree of freedom [6, 7]. In 1998, Bender with Boettcher [8] proposed an alternative mathematical interpretation of the phenomenon. They pointed out that within the conventional Hermitian formulations of quantum theory the quantitative description of the quantum phase transition phenomena is difficult, mainly because one has to interrelate the unitary quantum world with the non-unitary quantum world (cf. also the Jones' dedicated study [9]).

The latter observation served as an immediate inspiration of our present study. The paper will be organized as follows. In introductory section 21) we shall recall a few facts about $\mathcal{P} \mathcal{T}$-symmetry and about quantum phase transitions. In section 3 we then introduce, via a schematic model, the key concept of our paper, viz, the notion of the so called Hermitian-non-Hermitian interface. This material will be followed by sections 4 and 5 in which we explain that for our present purposes the stationary non-Hermitian Schrödingerpicture description of quantum dynamics of reviews [10, 11, 12] would not suffice, and that we shall need its generalized, time-dependent and hiddenly Hermitian versions as described in Refs. [13, 14] and as critically reviewed, more recently, in [15]. A compact summary of the theory will be provided, listing the dynamical equations in section 4 and then turning attention to some of the purely phenomenological aspects of the resulting picture of physics in section 5. In section 6 we then return again from the abstract theo- 
retical lesson to our concrete illustrative benchmark model. We use it to explain, in some technical detail, some overall features of the process of the abstract construction of the appropriate physical Hilbert space $\mathcal{H}^{(\text {second) }}$ and of its concrete representation (based on the mere amendment of the inner product) in the more friendly (albeit manifestly unphysical) Hilbert space $\mathcal{H}^{(\text {first })}$. The text will be then completed by the two shorter sections of Discussion and Conclusions.

\section{$2 \mathcal{P} \mathcal{T}$-symmetry and its breakdown}

In the literature devoted to quantum systems and to the questions of their stability theoreticians are usually clearly separating the conventional Hermitian theories (in which the energies are assumed real and in which the evolution is unitary) from the traditional versions of non-Hermitian theories which deal, exclusively, with unstable and resonant quantum systems. An explanation of such a split of scope may be found in chapter 10 of monograph [7]. The author's attention is paid there to the latter, complex-energy models. A parallel outline of the current understanding of the unitary, stable quantum systems may be sought, e.g., in the most recent collection of reviews [16]. In our present paper we shall restrict our attention just to the latter subclass of the quantum models and phenomena in which the energies remain real even if the representation of the observables themselves becomes non-Hermitian, viz., quasi-Hermitian [10] or $\mathcal{P} \mathcal{T}$-symmetric [11] or pseudo-Hermitian [12].

\subsection{Bound states: The loss of observability}

The main purpose of our present paper is a clarification of several paradoxes which were mentioned, in the literature, after the publication of the Bender's and Boettcher's influential letter [8]. The conventional Hermitian formulation of quantum mechanics has been declared there over-restrictive. The authors illustrated their idea via a hypothetical, manifestly non-Hermitian ordinary differential Hamiltonian

$$
H=H^{(B B)}(\delta)=-\frac{d^{2}}{d x^{2}}+x^{2}(\mathrm{i} x)^{\delta} \neq H^{\dagger}
$$

living, for $\delta \in(0,2)$ at least, in the entirely conventional Hilbert space $L^{2}(\mathbb{R})$. They came to the conclusion that in spite of the manifest non-Hermiticity of the operator its energy spectrum is real, discrete and bounded from below, i.e., potentially observable (rigorously, the conjecture has been proved in [17]). This property has been attributed to the $\mathcal{P} \mathcal{T}$-symmetry of the Hamiltonian where $\mathcal{P}$ means parity while the antilinear operator $\mathcal{T}$ simulates time-reversal [11]. 
At the beginning of the new millennium the resolution of the apparent contradiction between the manifest non-Hermiticity of the Bender's and Boettcher's Hamiltonian and the strict reality of the bound-state energies has been found in the older, quasi-Hermitian formulation of quantum mechanics [10, 12]. The idea has been made widely accepted, mostly under the name of $\mathcal{P} \mathcal{T}$-symmetric quantum mechanics (PTQM).

As a consequence of the subsequent developments, the modern theoretical description of the unitary quantum systems is now already partitioned into the so called Hermitian and non-Hermitian branches. The former branch is thoroughly explained in conventional textbooks [1]. For an introduction in the latter, PTQM-inspired philosophy the reader is recommended to consult, e.g., the well-written reviews [11, 12] (cf. also the non-stationary extension of the formalism as introduced in [13] and reviewed in [14]). It is worth emphasizing that only the proper use of the PTQM-inspired formulation of quantum theory endowed, e.g., the first-quantized Klein-Gordon equation of textbooks, almost a full century after its introduction, with a correct and consistent probabilistic interpretation [18].

From the pragmatic point of view of experimental physics one of the most important innovations characterizing the nonstandard PTQM models may be seen in their capability of reaching the very boundary of the unitary and stable dynamical regime. For example, the spectrum of the toy model (1) is real (i.e., in principle, compatible with the unitarity of the evolution) at all of the non-negative exponents $\delta \geq 0$. This spectrum, nevertheless, immediately loses these properties at an arbitrarily small negative $\delta<0$ where the reality of the energies only survives for a finite, $\delta$-dependent number of the low-lying levels [8].

In the extensive dedicated literature, the sudden loss of the stability of the system at certain parameters and couplings is usually interpreted as the spontaneous breakdown of $\mathcal{P} \mathcal{T}$-symmetry [11]. This loss may be interpreted as a quantum phase transition of the first kind [4] and/or as a cusp-like quantum catastrophe [19]. Various simulations of such an abrupt loss of the observability of the energy made the PTQM formalism also enormously popular among mathematicians [16] and non-quantum theoreticians and experimental physicists [20, 21].

\subsection{Scattering states: Giving up the unitarity}

During the birth of the PTQM formalism the conventional self-adjoint phenomenological Hamiltonians $\mathfrak{h}=\mathfrak{h}^{\dagger}$ (with the robust reality of the spectrum) were declared not sufficiently flexible. The innovated models sampled by Eq. (1) were found compatible with the above-mentioned hypothetical, phenomenologically well motivated requirement of the possibility of a merger and of a subsequent complexification of the energy eigenvalues. In the context of physics the phenomenon of such a type has been interpreted as a spontaneous 
breakdown of the $\mathcal{P} \mathcal{T}$-symmetry of the system (see, e.g., the physics-oriented review [11] for details). The mathematical essence of the necessary generalization of the formalism has been reconfirmed to lie in the non-Hermiticity of the operators with real spectra. In the benchmark model (1) even the technical constraint $\delta \in(0,2)$ has been found removable. After an ad hoc, $\delta$-dependent amendment of the integration contour of $x$ the spectrum has been shown to stay real for all of the non-negative real exponents $\delta \in[0, \infty)$.

The loss of the reality of the spectrum occurred at $\delta=0$. It was not too difficult to conclude that the evolution controlled by the toy-model Hamiltonian (1) is deeply nonunitary at $\delta<0$. A more sophisticated interpretation of the evolution at $\delta \in(0,2)$ or at $\delta \in(0, \infty)$ was needed of course, but the necessary amendment of the theory has been developed soon. In brief, the evolution has been found unitary in an ad hoc Hilbert space $\mathcal{H}^{(\text {physical })} \neq L^{2}(\mathbb{R})$ in which the Hamiltonian itself (which is non-Hermitian in $L^{2}(\mathbb{R})$ where we write $H \neq H^{\dagger}$ ) is reinterpreted as self-adjoint [12].

One of the limitations of the applicability of the PTQM approach was revealed by Jones [22]. He turned attention to the dynamical regime of unitary quantum scattering and replaced the bound-state Hamiltonian (1) by the point-interaction toy model

$$
H=H^{(H J)}(\alpha, \beta, L)=-\frac{d^{2}}{d x^{2}}+\alpha V_{(H)}(x)+\mathrm{i} \beta V_{(N)}(x, L)
$$

The conventional attractive delta-function interaction $V_{(H)}(x)=-\delta(x)$ [which is Hermitian in $L^{2}(\mathbb{R})$ ] was complemented there by a non-Hermitian but $\mathcal{P} \mathcal{T}$-symmetric partner $V_{(N)}(x, L)=\delta(x-L)-\delta(x+L)$. After a detailed analysis of the model the Jones' ultimate recommendations were discouraging. He came to the conclusion that one cannot accept the fact that in the non-Hermitian picture the scattering in-state waves "enter from both left and right" and that there exists no Hermitian/non-Hermitian-interaction interface, i.e., in his interpretation, a spatial separation distance $L \approx L_{0}$ at which one could treat model (2) as both quasi-Hermitian and purely Hermitian. In his own words, "the physical picture changes drastically when going from one picture to the other" so that "the only satisfactory resolution of this dilemma is to treat the non-Hermitian scattering potential as an effective one, and work in the standard framework of quantum mechanics, accepting that this effective potential may well involve the loss of unitarity when attention is restricted to the quantum mechanical system itself and not its environment" 9].

\section{Phase transitions in a benchmark model}

In contrast to the abrupt and drastic physics-changing losses of observability and/or of causality as mentioned above, the more subtle and "softer", energy-conserving quantum 
phase transitions of the second kind do not seem to have attracted the attention of the experimentalists yet. We believe that such an attention could be re-attracted by our forthcoming conceptual considerations.

A rarely emphasized theoretical possibility of the energy-conserving phase-transition processes of the second kind has already been noticed to exist in several less popular $\mathcal{P} \mathcal{T}$-symmetric toy models [23, 24, 25]. Here, we are reopening the discussion. We are persuaded that, in particular, the Jones' scepticism is mathematically correct but that it is based, in the context of physics, on a rather subtle misunderstanding. At an arbitrary fixed set of parameters, indeed, the quasi-Hermitian and Hermitian pictures of reality must be, by definition, strictly equivalent [12]. In other words, the concept of a Hermitian/nonHermitian-interaction interface alias Hermitian-quasi-Hermitian phase transition can only be introduced as a specific, model-dependent set of parameters $\mathcal{D}_{\text {(interface) }}$ at which the Hermitian and quasi-Hermitian representations of a quantum system would coincide [26].

\subsection{The existence of interface}

According to the conventional, Hermitian quantum theory of textbooks the parameterand time-dependent family of matrices

$$
\mathfrak{h}(c, t)=\left[\begin{array}{cc}
-1 & \mathrm{i} \sqrt{t^{2}-c} \\
-\mathrm{i} \sqrt{t^{2}-c} & 1
\end{array}\right]
$$

can be perceived as an elementary sample of a phenomenological Hamiltonian representing a stable quantum system $\mathcal{S}_{\text {(conventional) }}$ if an only if the matrix is Hermitian in the preselected physical Hilbert space, i.e., say, in $\mathcal{H}_{(\text {conventional })}=\mathbb{C}^{2}$,

$$
\mathfrak{h}(c, t)=\mathfrak{h}^{\dagger}(c, t), \quad t^{2}-c \geq 0
$$

The evolution of the underlying quantum system will be unitary due to the Stone theorem [27]. In Schrödinger picture this evolution will be controlled by the conventional Schrödinger equation

$$
\mathrm{i} \frac{d}{d t}|\psi(t) \succ=\mathfrak{h}(c, t)| \psi(t) \succ, \quad \mid \psi(t) \succ \in \mathcal{H}_{(\text {conventional })} .
$$

In the plane of parameters $c$ and $t$ the set of admissible values

$$
\mathcal{D}_{(\text {conventional })}=\left\{(c, t) \mid c \leq t^{2}\right\}
$$

will fill the space on, and below, the lower, thicker parabola of Figs. 1 and 2 , 


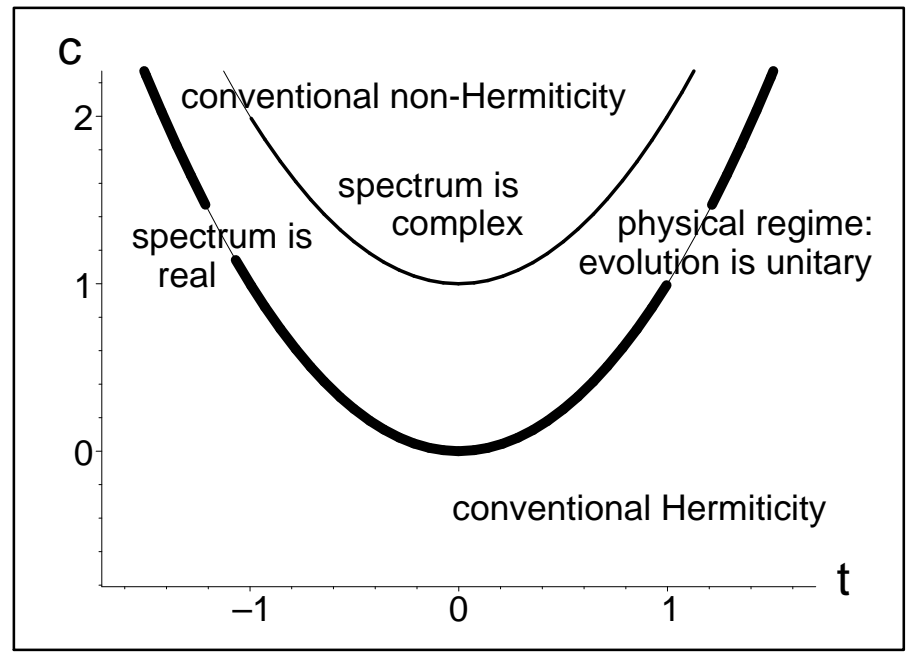

Figure 1: The loss of the conventional Hermiticity with the growth of $c$ across the thick curve, and the ultimate end of the observability of the energy in $c-t$ plane (the spontaneous breakdown of $\mathcal{P} \mathcal{T}$-symmetry, thin curve) for toy-model Hamiltonian (3).

According to the slightly less conventional versions of quantum theory (cf. review [12]) the candidates (3) for Hamiltonians may be made phenomenologically acceptable whenever the energies remain real and non-degenerate, i.e., potentially observable. Thus, after an ad hoc amendment of the physical Hilbert space the conventional Hermiticity requirement can be generalized and replaced by a more sophisticated but less restrictive hidden Hermiticity called quasi-Hermiticity [28]. For our matrix (3) such an innovation of the theory would imply that the unitarity of the evolution of the underlying quantum system can be guaranteed even in a non-Hermitian dynamical regime or, more precisely, whenever the easily evaluated eigenvalues

$$
E_{ \pm}(c, t)= \pm \sqrt{t^{2}+1-c}
$$

satisfy the much weaker reality and non-degeneracy constraint. Besides the above-mentioned "Hermitian" quantum systems $\mathcal{S}_{(\text {conventional })}$ one can, therefore, speak also about the nonHermitian but still unitary quantum systems $\mathcal{S}_{(\text {quasi-Hermitian })}$.

\subsection{Quasi-Hermitian regime}

In the light of Eq. (6) the quasi-Hermitian extension of the scope of quantum theory is feasible if and only if the parameters $c$ and $t$ stay confined inside a complementary open set of admissible parameters,

$$
\mathcal{D}_{(\text {quasi-Hermitian })}=\left\{(c, t) \mid t^{2}<c<t^{2}+1\right\} .
$$




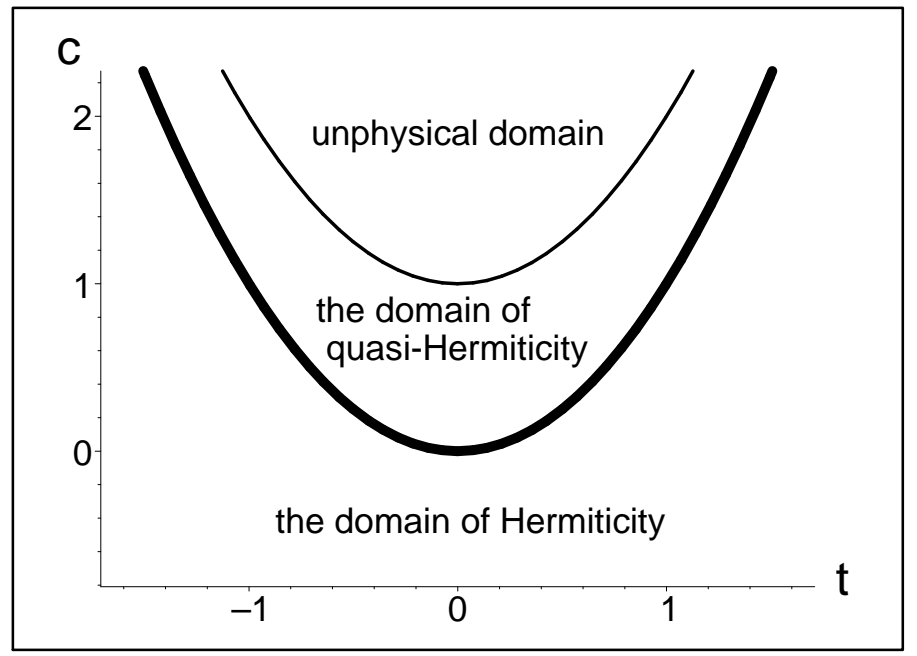

Figure 2: Hermitian-quasi-Hermitian interface (thick curve, Eq. (88)).

In Figs. 1 and 2 such a "quasi-Hermiticity" domain of the new eligible parameters fills the curved-stripe space between the two parabolas. The thicker parabolic curve lies in the middle of the phenomenologically admissible physical domain

$$
\mathcal{D}_{(\text {admissible })}=\mathcal{D}_{(\text {conventional })} \bigcup \mathcal{D}_{(\text {quasi-Hermitian })}
$$

which fills the whole space below the thinner parabola. Obviously, the lower, thick curve represents a well defined boundary between the Hermitian (= lower) and quasi-Hermitian (= upper) unitary-evolution regimes. The Hamiltonian itself is diagonal along this curve. We will call this curve an "interface",

$$
\mathcal{D}_{(\text {interface })}=\left\{(c, t) \mid c=t^{2}\right\}
$$

We shall also slightly change here a few other notation conventions. Firstly, our toy model Hamiltonian will exclusively be written in the lower-case format of Eqs. (3) + (44) on and below the interface curve (8) of Figs. 1 and 2. In parallel, the same matrix will be always denoted by the upper-case symbol whenever the parameters get chosen above the interface, making the matrix non-Hermitian,

$$
H(c, t)=\left[\begin{array}{cc}
-1 & \sqrt{c-t^{2}} \\
-\sqrt{c-t^{2}} & 1
\end{array}\right] \neq H^{\dagger}(c, t), \quad c>t^{2} .
$$

The key purpose of such a restriction is to underline that the physics behind the different symbols (viz., behind $\mathfrak{h}(c, t)$ with $c<t^{2}$ and behind $H(c, t)$ with $c>t^{2}$ ) is different. For two reasons. Not only because the parameters are different but, first of all, because in contrast 
to $H(c, t) \neq H^{\dagger}(c, t)$, the self-adjoint operator $\mathfrak{h}(c, t)$ may be assigned the conventional spectral representation (cf. the account of such an aspect of the theory in [29]).

Let us conclude that we do not need to care about the description of the system in the Hermitian dynamical regime. This would be purely routine and the details can be left to the reader. In contrast, after the system passes the interface and enters the quasi-Hermitian domain, multiple technical aspects of its description become far from trivial.

\section{Non-stationary quasi-Hermitian dynamics in nuce}

We shall need the time-dependent extension of the stationary PTQM formalism of Refs. [11, 12 in the form which has been proposed in Ref. [13]. The name of "three-Hilbert-space (3HS) formulation of quantum mechanics" was coined and advocated for this upgrade of the theory in subsequent compact reviews [14, 15, 30]. Nontrivial applications of the resulting non-stationary 3HS approach are currently being sought [30, 31, 32, 33, 34]. Attention is being shifted from the stationary context to the general time-dependent scenario in which the quasi-Hermitian (i.e., in our notation, upper-case) and time-dependent generic Hamiltonians $H(t)$ are treated as isospectral to their Hermitian (i.e., in our notation, lower-case) alternative representatives

$$
\mathfrak{h}(t)=\Omega(t) H(t) \Omega^{-1}(t)=\mathfrak{h}^{\dagger}(t)
$$

In our present study of matrix models, the ad hoc construction of a suitable invertible (often called Dyson's) map $\Omega(t)$ is just a routine linear-algebraic procedure. As long as relation (10) may be rearranged to read

$$
H^{\dagger}(t) \Theta(t)=\Theta(t) H(t), \quad \Theta(t)=\Omega^{\dagger}(t) \Omega(t)
$$

the matrix operator $H(t)$ may be declared quasi-Hermitian whenever we manage to find $\Omega(t)$ and $\Theta(t)$ such that Eq. (11) is satisfied.

\subsection{The doublet of Schrödinger equations}

In Refs. [14, 15] the ultimate, non-stationary 3HS version of the quasi-Hermitian quantum theory is characterized as a representation of a quantum system $\mathcal{S}_{(\text {quasi-Hermitian) }}$ which is based on the simultaneous use of the three representative Hilbert spaces $\mathcal{H}^{(\text {first })}, \mathcal{H}^{(\text {second })}$ and $\mathcal{H}^{(\text {conventional })}$. The ket-vectors $|\psi\rangle \in \mathcal{H}^{(\text {first })}$ are assumed to coincide with the kets $|\psi\rangle \in \mathcal{H}^{(\text {second })}$. In the nuclear-physics exemplification [10] both of them describe the "effective" bosons while the "real" nucleons, fermions, have to be represented by different, spiked-ket symbols $\mid \psi \succ \in \mathcal{H}^{\text {(conventional) }}$. 
The mutual correspondence

$$
|\psi(t) \succ=\Omega(t)| \psi(t)\rangle .
$$

between the kets is just a time-dependent generalization of the Dyson's old idea [13, 35]. In its spirit one inserts ansatz (12) in the conventional lower-case Schrödinger equation sampled by Eq. (5) which lives in the Hilbert space $\mathcal{H}^{(\text {conventional })}$ of textbooks. This insertion leads to the equivalent equation

$$
\mathrm{i} \frac{d}{d t}|\psi(t)\rangle=G(t)|\psi(t)\rangle
$$

which is defined in both of the spaces $\mathcal{H}^{(\text {first })}$ and $\mathcal{H}^{(\text {second })}$. We must only add that

$$
G(t)=H(t)-\Sigma(t), \quad \Sigma(t)=\mathrm{i} \Omega^{-1}(t)\left[\frac{d}{d t} \Omega(t)\right] .
$$

We have to remind the readers that our instantaneous-energy-operator $H(t)$ is assumed to be defined in a "friendly" Hilbert space $\mathcal{H}^{(\text {first })}$ in which it is non-self-adjoint even though it possesses the real (i.e., in principle, observable) spectrum. This is the reason why another, "sophisticated" Hilbert space $\mathcal{H}^{(\text {second })}$ had to be introduced:

- the space $\mathcal{H}^{(\text {second) }}$ is physical - it is constructed as unitarily equivalent to $\mathcal{H}^{(\text {conventional })}$, i.e., to the Hilbert space of textbooks;

- the conventional space is, by assumption, "prohibitively complicated" and useless [10, 35]. Simplifications are expected from our working in $\mathcal{H}^{(\text {second) }}[12]$;

- the manifestly unphysical Hilbert space $\mathcal{H}^{(f i r s t)}$ is assumed friendly. It is, therefore, used as a carrier of the representation of $\mathcal{H}^{(\text {second) }}$ (realized via a modification of the inner product).

Conceptually, the latter representation is easy. It merely requires a replacement of the antilinear Hermitian conjugation defining the first space,

$$
\mathcal{T}^{(\text {first })}:|\psi\rangle \rightarrow\langle\psi| \in\left(\mathcal{H}^{(\text {first })}\right)^{\prime}
$$

by its second-space bra-to-curlyket analogue

$$
\mathcal{T}^{(\text {second })}:|\psi\rangle \rightarrow\left\{\psi \mid \in\left(\mathcal{H}^{(\text {second })}\right)^{\prime} .\right.
$$

For this purpose it is sufficient to postulate the identification

$$
\left.|\psi(t) \succ=\Omega(t)| \psi(t)\rangle=\left[\Omega^{\dagger}(t)\right]^{-1} \mid \psi(t)\right\} .
$$


One of the most immediate consequences is the validity of the conjugate-evolution Schrödinger equation

$$
\left.\left.\mathrm{i} \frac{d}{d t} \mid \psi(t)\right\}=G^{\dagger}(t) \mid \psi(t)\right\}
$$

Another, equally useful consequence is that the conventional textbook orthogonality and/or

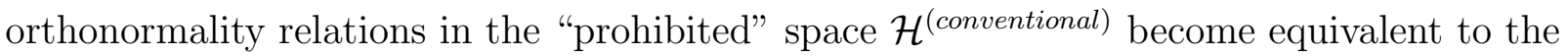
biorthogonality and/or biorthonormality relations in the "recommended" space $\mathcal{H}^{(\text {second })}$,

$$
\prec \psi_{1} \mid \psi_{2} \succ=\left\{\psi_{1}\left|\psi_{2}\right\rangle\right. \text {. }
$$

Once the latter space is always represented in the friendly, auxiliary Hilbert space $\mathcal{H}^{(\text {first })}$, one simply defines $\mid \psi\}=\Theta|\psi\rangle[14]$.

\subsection{The doublets of Heisenberg equations}

The variability of the Dyson maps with time forces us to realize that even if the observable quantity represented by a lower-case operator $\lambda$ is chosen time-independent, its isospectral partner would vary with time, anyhow. Still, in [36] we revealed that the work with the non-stationary lower-case operators is truly tedious so that we will keep assuming here that $\lambda=\lambda^{\dagger} \neq \lambda(t)$ in $\mathcal{H}^{(\text {conventional })}$. Then we may Dyson-map

$$
\lambda \rightarrow \Lambda(t)=\Omega^{-1}(t) \lambda \Omega(t) .
$$

The straightforward differentiation of this product leads to the operator differential equation of Heisenberg type,

$$
\mathrm{i} \frac{d}{d t} \Lambda(t)=\Lambda(t) \Sigma(t)-\Sigma(t) \Lambda(t) .
$$

Again, the self-adjointness in $\mathcal{H}^{(\text {conventional })}$ is translated into the quasi-Hermiticity in $\mathcal{H}^{(\text {first })}$,

$$
\Lambda^{\dagger}(t)=\Theta(t) \Lambda(t) \Theta^{-1}(t)
$$

Whenever we wish to keep the trace of the observability explicit, it makes sense to work, in parallel, with the second Heisenberg equation

$$
\mathrm{i} \frac{d}{d t} \Lambda^{\dagger}(t)=\Lambda^{\dagger}(t) \Sigma^{\dagger}(t)-\Sigma^{\dagger}(t) \Lambda^{\dagger}(t)
$$

In the special case of the observable energy $H(t)$ in the conservative scenario with $\mathfrak{h} \neq \mathfrak{h}(t)$ the pair of Eqs. (21) and (23) could be also used and, in this case, modified,

$$
\mathrm{i} \frac{d}{d t} H(t)=G(t) H(t)-H(t) G(t) .
$$


An independent comment can be made concerning the metric operator for which one starts from the elementary mathematical identity

$$
\mathrm{i} \frac{d}{d t} \Theta(t)=\Theta(t) \Sigma(t)-\Sigma^{\dagger}(t) \Theta(t)
$$

and deduces its equivalent alternative

$$
\mathrm{i} \frac{d}{d t} \Theta(t)=G^{\dagger}(t) \Theta(t)-\Theta(t) G(t) .
$$

In recent paper [15] the direct solution of Eq. (26) was discouraged as tedious, inefficient and not always necessary. Still, one could try to solve this differential equation for operator $\Theta(t)$, numerically, in some sufficiently elementary special cases. This was done, e.g., by Hynek Bíla [37] and, later, by several other teams of researchers [34. All of these authors revealed and pointed out that the resulting operators of the Hilbert space metric $\Theta(t)$ seem to be enormously sensitive not only to the properties of the generators $G(t)$ but also to the initial choice of $\Theta(t)$ at the preparation time, i.e., in our present physical context, at the interface, i.e., at the instant $t=t_{0}$ of the phase transition.

\section{Physics of quasi-Hermitian Hamiltonians in nuce}

In our present model-based analysis of the phenomena connected with the existence of the Hermitian-quasi-Hermitian interface we may follow the conventional textbooks and use trivial $\Omega(c, t)=I$ whenever $(c, t) \in \mathcal{D}_{\text {(conventional) }}$, i.e., in the Hermitian regime. In the more sophisticated regime with $(c, t) \in \mathcal{D}_{\text {(quasi-Hermitian) }}$, a non-trivial $\Omega(c, t) \neq I$ will be needed. After such a generalization the formalism becomes perceivably more complicated.

\subsection{Terminology}

The information about the (say, unitary) time-evolution of a given quantum system $\mathcal{S}$ can be carried by its wave function $\psi(t)$ (one then speaks about the Schrödinger picture of the reality, SP [38]), or by the relevant observables $\mathfrak{q}(t)$ (in the so called Heisenberg picture, HP [39]), or both (in the universal Dirac's alias interaction picture, IP [1]). In the light of the recent theoretical developments (cf., e.g., dedicated book [16]) one can further distinguish between the so called Hermitian and non-Hermitian versions of the respective alternative formulations of the quantum dynamical laws. Thus, the traditional reviews of the Hermitian formulations (e.g., [40]) may be complemented by the detailed outlines of the non-Hermitian Schrödinger picture (NSP, [11, 12]), of the non-Hermitian Heisenberg picture (NHP, [36, 41]) and/or of the non-Hermitian interaction picture (NIP, [13, 14, 15, 42]). 
The shared feature of all of the innovative non-Hermitian pictures is that they work with the operators of observables (say, $Q(t)$ ) which are only non-Hermitian in an auxiliary, mathematically strongly preferable and technically friendly but plainly unphysical Hilbert space $\mathcal{H}^{\text {(first) }}$. In this sense the widespread use of the term "non-Hermitian operators" (so that one writes $Q(t) \neq Q^{\dagger}(t)$ ) is mathematically correct (in $\mathcal{H}^{(\text {first })}$ ) but conceptually misleading. This is because all of our "exotic" observables $Q(t)$ may be reinterpreted as traditional and self-adjoint immediately after one replaces the auxiliary, "false" Hilbert space $\mathcal{H}^{(\text {first })}$ by its manifestly physics-representing "standard" amendment $\mathcal{H}^{(\text {second })}$.

The survival of the misleading terminology had a few pragmatic and/or historical reasons. The main one is that the "correct" physical Hilbert space $\mathcal{H}^{(\text {second })}$ is in fact never used in practice. In the majority of applications it is either represented in $\mathcal{H}^{(\text {first })}$ (see the preceding section) or replaced by its unitarily equivalent avatar $\mathcal{H}^{(\text {conventional })}$. In the former case one should better write, e.g., $Q(t)=Q^{\ddagger}(t):=\Theta^{-1}(t) Q^{\dagger}(t) \Theta(t)$.

In the literature, the notation is far from being unified. For example, in the stationary quasi-Hermitian formalisms, our present symbol $\Theta$ (denoting the special, time-independent physical Hilbert-space metric) is just a Greek translation of the symbol $T$ used in the oldest review [10]. For the same operator, an entirely different, subscripted symbol $\eta_{+}$was introduced by Mostafazadeh [12]. Equivalently, people also use the special $\Theta$ s equal to the products $\mathcal{P C}$ of parity with the (Hamiltonian-dependent) charge [11].

\subsection{Measurements}

From the point of view of experimentalists, the evolution of any quantum system controlled by the equations of preceding section must be initiated by the preparation of the system (say, in a pure state) at an "initial" time (say, at $t=0$ ). Subsequently, the verification of the predictions is to be performed using the measurement over the system at a suitable "final" time $t=T>0$. It is well known that "before the phase transition", i.e., in the conventional Hermitian regime the matrices $\Omega(c, t)=\Theta(c, t)=I$ may be kept trivial so that, in the 3HS language, all three Hilbert spaces coincide and the upper- and lowercase Hamiltonians are allowed to coincide as well, $\mathfrak{h}(c, t)=H(c, t)$. This means that in the conventional dynamical regime one just follows the textbooks. The predictions concerning, say, a time-dependent observable $\mathfrak{q}(t)$ are simply obtained via the routine evaluation of the mean-value overlaps

$$
\prec \psi(T)|\mathfrak{q}(T)| \psi(T) \succ
$$

where the time-dependence of the operator $\mathfrak{q}(t)$ is assumed prescribed in advance and where the time-dependence of the wave functions $\mid \psi(t) \succ$ is obtained by the solution of Schrödinger Eq. (15). 
After the quantum system in question passes the Hermitian-quasi-Hermitian interface and after it continues evolving in its quasi-Hermitian phase, the latter formula defined in $\mathcal{H}_{\text {(conventional) }}$ may be declared intractable because we are only given now our Hamiltonian (i.e., its toy model sample $H(c, t))$ in its non-Hermitan form (cf. Eq. (9)). Our overall methodical assumptions force us to use the general $3 \mathrm{HS}$ formalism and, in particular, the nontrivial forms of the Dyson maps $\Omega(t) \neq I$. Also just the upper-cases representations of the observables may be assumed tractable. In the light of Eqs. (10) and (20) (in its specification $\left.Q(t)=\Omega^{-1}(t) \mathfrak{q}(t) \Omega(t)\right)$ this means that the lower-case representatives of the observables become unknown and different from their upper-case avatars. Fortunately, in the general 3HS setting the knowledge the lower-case observables is superfluous due to the identity

$$
\prec \psi(T)|\mathfrak{q}(T)| \psi(T) \succ=\{\psi(T)|Q(T)| \psi(T)\rangle .
$$

Thus, it is sufficient to evaluate just the right-hand-side expression in practice.

\subsection{Instantaneous energies}

Via Figs. 1 and 2 we discussed, in section 3 , the influence and the consequences of the growth of parameter $c$ at a given time $t$. For the purposes of the study of dynamics in the language of the above-outlined non-stationary $3 \mathrm{HS}$ formalism it makes better sense to keep the parameter $c$ unchanged and to check what is happening during the evolution of the system in time.
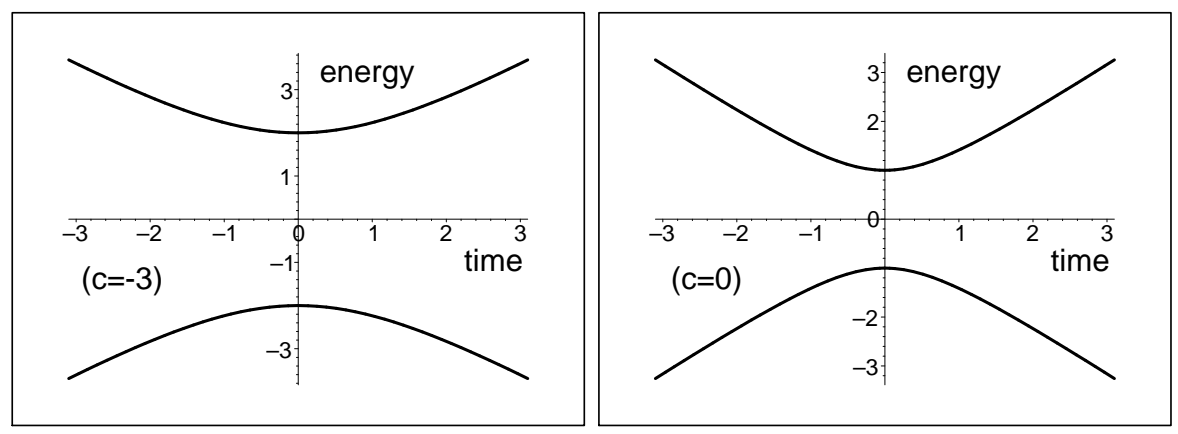

Figure 3: Constant-c energies $E_{ \pm}(c, t)$ of Eq. (6) in Hermitian regime (left picture) and on its boundary (right picture).

What is of primary interest is the time-dependence of the (in principle, measurable) instantaneous energies as prescribed by Eq. (6) . In the two pictures of Fig. 3 let us sample the conventional scenario in which the energies exhibit a characteristic pattern of the so called "avoided crossing". 


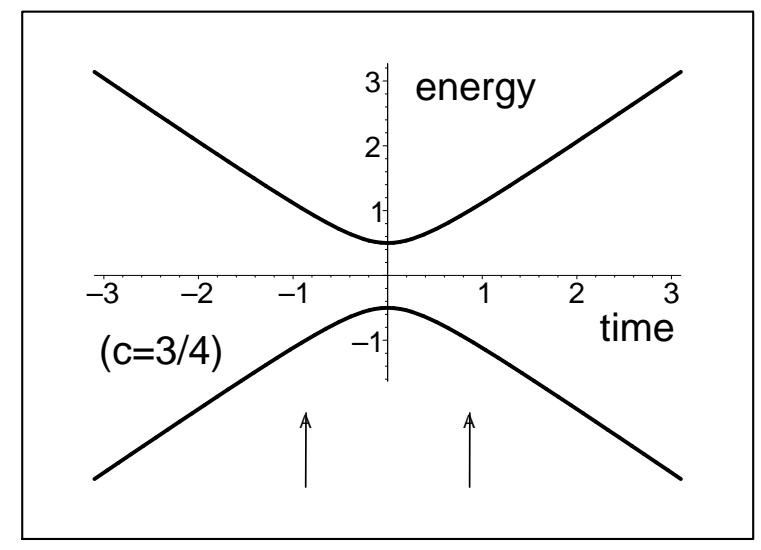

Figure 4: Constant-c energies (6) in the partially quasi-Hermitian regime. The two arrows mark the interval of non-Hermiticity with boundaries $t_{(\text {interface })}= \pm \sqrt{c} \approx \pm 0.866$.

In the subsequent example of Fig. 4 we see that the same avoided crossing behavior remains unchanged even if we move to a non-Hermitian but still quasi-Hermitian dynamical regime with $c \in(0,1)$. From the spectrum itself one could hardly guess that a nontrivial Hilbert-space metric $\Theta(t) \neq I$ must be constructed in the interval of $t \in(-\sqrt{c}, \sqrt{c})$. Inside this interval we have $(c, t) \in \mathcal{D}_{\text {(quasi-Hermitian) }}$ so that the probabilistic interpretation of the non-stationary quantum system in question becomes nontrivial. The predictions of the results of instantaneous measurements must be calculated using the right-hand-side expression in formula (28) of course. In other words, besides the necessity of the solution of the pair of Schrödinger Eqs. (13) and (18), also the time-dependence of the generic observables must be deduced from the solution of the underlying Heisenberg-like equations as discussed in Ref. [15] in full detail, and as sampled in subsection 4.2 above.
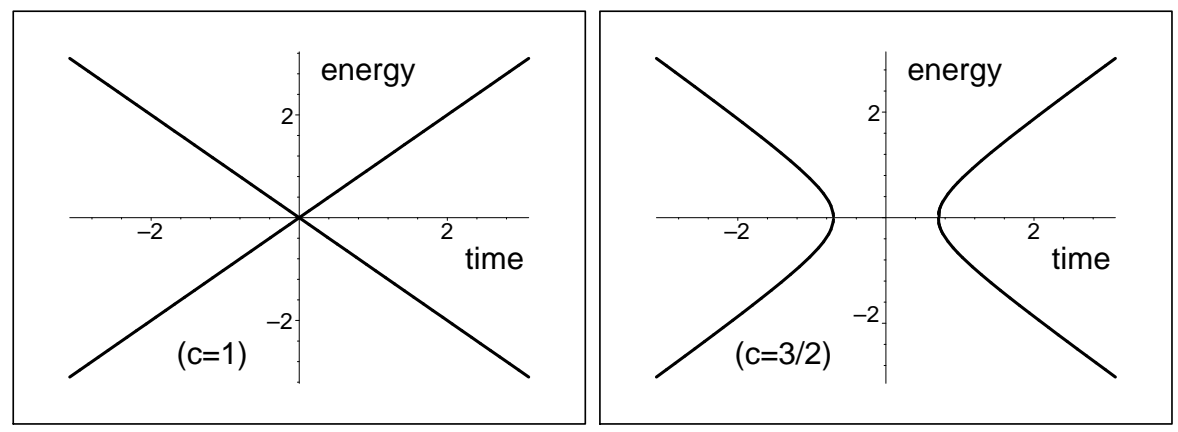

Figure 5: Real parts of the constant-c energies (6) at the boundary of the quasi-Hermitian regime (left picture: notice the degeneracy at $t=0$ ) and beyond (right picture).

We saw that in our toy models of paragraph 3.1 the avoided-crossing behavior of the 
spectrum is shared by the negative-parameter regime with $c<0$ (where the Hamiltonian is safely Hermitian) and by the partially quasi-Hermitian regime at the small and positive $c \in(0,1)$. The purely Hermitian description only survives there at such times that $t^{2}>c$, cf. Fig. 11. In contrast, a drastic qualitative change of the spectrum occurs when we choose a larger value of $c \geq 1$. This is illustrated by Fig. 5 where the left picture with $c=1$ samples the rather remarkable "unavoided-crossing" spectral anomaly, and where the right picture samples the $c>1$ scenario in which the energies cease to be real in the interval of $t \in(-\sqrt{c-1}, \sqrt{c-1})$.

The two instants $t_{ \pm}= \pm \sqrt{c-1}$ of the "quantum catastrophe" [19] mark the collapse of the system and reflect the spontaneous breakdown of its $\mathcal{P} \mathcal{T}$-symmetry [11]. In mathematics these values are called "exceptional points" [43]. Inside the interval of $t \in(-\sqrt{c-1}, \sqrt{c-1})$ our toy model Hamiltonian $H(c, t)$ of Eq. (9) is not even quasiHermitian. It ceases to describe any physical reality whatsoever. In contrast, once we restrict attention, say, to the left half-line of time $t \in(-\infty,-\sqrt{c-1}$, the importance of the Hermitian-to-non-Hermitian interface (8) is enhanced because its existence now represents a gate and one of conditions of the realization of the evolution leading to an ultimate fall of the quantum system into instability. This makes the models with $c>1$ theoretically relevant, phenomenologically appealing and methodically truly interesting.

\section{Benchmark model: Probabilistic interpretation}

Whenever we are given the operators of observables and whenever we find the metric $\Theta$ compatible with relations (22), we may factorize $\Theta \rightarrow \Omega$ and formulate the dynamical evolution equations. The recipe (cf. Ref. [15]) has thoroughly been described above. For its present new application let us now return to matrix (9) with parameters $c$ (coupling) and/or $t$ (time) localized, in the $c-t$ plane, between the two parabolic curves of Fig. 1 , Once we assume that the parameters lie not too far from the lower parabola (i.e., from the interface of our current interest), matrix (9) can be perceived as the operator of an observable energy. It characterizes our hypothetical quantum system which was initially Hermitian but which suffered the phase transition. This means that the parameters were slightly changed. The system passed the Hermitian-quasi-Hermitian interface but in the new dynamical regime the evolution is still unitary. 


\subsection{Metric operator}

In the quasi-Hermitian regime the physical contents of our real Hamiltonian matrix $H(c, t)$ of Eq. (9) is given by the real and symmetric matrix

$$
\Theta(c, t)=\left[\begin{array}{ll}
a(c, t) & b(c, t) \\
b(c, t) & d(c, t)
\end{array}\right]=\Theta^{\dagger}(c, t)>0
$$

of the Hilbert-space metric. Compatibility condition (11) may be checked to hold if and only if $b(c, t)=\gamma(c, t) u(c, t)$ where $\gamma(c, t)=\sqrt{c-t^{2}}$ and $u(c, t)=[a(c, t)+d(c, t)] / 2$. In this notation the energy eigenvalues (6) acquire the transparent form $E_{ \pm}=\sqrt{1-\gamma^{2}(c, t)}$ so that with $\gamma(c, t)=\sin \tau(c, t)$ the whole information about our quasi-Hermitian input Hamiltonian is reduced to the specification of $\tau(c, t) \in(0, \pi / 2)$. Effectively, this parameter measures the distance from the interface (8)).

What remains for us to guarantee is the positivity of the metric. This means that with $v(c, t)=a(c, t) d(c, t)$ both of its eigenvalues $\theta_{ \pm}=u \pm \sqrt{\left(1+\gamma^{2}\right) u^{2}-v}>0$ must remain real (i.e., we must have $v \leq\left(1+\gamma^{2}\right) u^{2}$ ) and positive (i.e., we must require $u>0$ and $v>\gamma^{2} u^{2}$ ). This enables us to reparametrize $v=v(u, \xi)=\gamma^{2} u^{2}+u^{2} \cos ^{2} \xi$ with $\xi \in(0, \pi / 2)$. The change $v \rightarrow \xi$ also simplifies $\theta_{ \pm}=(1 \pm \sin \xi) u$. Thus, the entirely general form of the metric will vary with the two free parameters, viz., with $u=u(c, t) \in(0, \infty)$ and with $\xi=\xi(c, t) \in(0, \pi / 2)$.

The backward changes of parameters yielding the explicit form of metric (29) are trivial: The derivation of the formulae is left to the readers. With this being done, the first step of the construction of the model would be completed. What would have to follow in applications would be the factorization of the metric into Dyson maps $\Omega(c, t)$, the construction of operators $\Sigma(c, t)$ and $G(c, t)$ (cf. Eq. (14)) and, finally, the solution of Schrödinger and Heisenberg equations.

\subsection{Physics near the interface}

One of the key messages delivered by the preceding subsection is that due to the nonstationarity of our toy model Hamiltonian $H=H(c, t) \equiv H[\gamma]$ one can select its physical interpretation out of a two-parametric menu of the eligible metrics $\Theta=\Theta(\gamma, u, \xi)$, i.e., of the physical Hilbert spaces $\mathcal{H}^{(\text {second })}(\gamma, u, \xi)$, i.e., of the metric-dependent sets of the quasi-Hermitian operators of observables $\Lambda=\Lambda_{j}(\gamma, u, \xi)$ with $j=1,2, \ldots$ [cf. Eq. (22)].

The concrete specification of our present quasi-Hermitian model as a system which was created by its passage through the interface has two important methodical consequences. Firstly, it makes sense to simplify our task and to restrict the scope of our analysis to the 
very small vicinity of the interface, i.e., to the very small (though still positive) values of the dynamical input-representing parameter $\gamma \ll 1$, i.e., of the re-scaled time initiated as the instant of the phase transition. Secondly, we have to postulate that all of the changes of the system with time should be smooth. In particular, this means that the two eigenvalues $\theta_{ \pm}=(1 \pm \sin \xi) u$ of the metric should be smooth functions of $\gamma$, i.e., the difference $u-1$ and the size of the second parameter $\xi$ should remain small at the small "re-scaled times" $\gamma \ll 1$.

Due to the elementary two-by-two matrix nature of our present benchmark example we can make use of the available explicit formulae and we could easily deduce the explicit forms of the corresponding illustrative power-series expansions. It is methodically more important to notice that the explicit construction of the approximations can proceed, in fact, in an entirely model-independent manner. One only has to consider a generic quasi-Hermitian $\gamma$-dependent Hamiltonian (i.e., say, its arbitrary non-Hermitian $N$ by $N$ real-matrix exemplification with real eigenvalues) which is defined, near the interface, by its Taylor series,

$$
H[\gamma]=H[0]+\gamma H^{\prime}[0]+\mathcal{O}\left(\gamma^{2}\right) .
$$

On the interface we have $H[0]=H^{\dagger}[0]$ of course. We can combine this general dynamical input information with a parallel perturbation-series ansatz for the related metric near the interface,

$$
\Theta[\gamma]=I+\gamma K+\mathcal{O}\left(\gamma^{2}\right)
$$

After insertion in the Dieudonné's compatibility condition (11) this will yield the first-order perturbation version of the constraint,

$$
H^{\dagger}[0] K-K H[0]=H^{\prime}[0]-\left(H^{\prime}[0]\right)^{\dagger}
$$

Routinely, this equation is to be solved, for $K$, by a suitable linear algebraic algorithm. In similar spirit one could also proceed in the higher-order perturbation constructions (advised by the referee we relocated these technicalities to a more mathematically oriented future publication).

\section{Discussion}

\subsection{Ambiguities}

In the present application of the non-stationary quasi-Hermitian theory we were only given the observable of energy in its manifestly non-Hermitian matrix representation (9). This implies that the Dieudonné's Eq. (22) can only be interpreted as a mere self-consistent 
restriction upon our choice of operators (say, of $\mathfrak{h}(c, t)$ and $\Omega(c, t)$ ) rather than as their unambiguous specification. This type of ambiguity was thoroughly discussed in Ref. [10]. In the context of physics the most elementary method of the removal of the ambiguity of the specification of operators $\mathfrak{h}(c, t)$ and $\Omega(c, t)$ may be based on certain additional phenomenological assumption. Besides the observability of the energy we may also require the existence of another (generic) observable represented, say, by a self-adjoint operator $\mathfrak{q}(c, t)=\mathfrak{q}^{\dagger}(c, t)$ [or operators, not necessarily $(c, t)$-dependent] or by its/their upper-case isospectral quasi-Hermitian avatar(s)

$$
Q(c, t)=\Omega^{-1}(c, t) \mathfrak{q}(c, t) \Omega(c, t)
$$

The mapping $\Omega(c, t)$ itself remains the same as before. This means that the Hermiticities of the lower-case operators can be simply reinterpreted as the respective Dieudonné's [28] quasi-Hermiticity properties (22).

\section{$7.2 \quad$ Interfaces}

Quantum phase transitions are usually interpreted as a breakdown of the unitarity of the evolution which is connected, in the light of the well known Stone's theorem [27], with an abrupt change of the effective Hamiltonian, i.e., with the sudden emergence of some new relevant degrees of freedom. Still, there exist the quantum evolution processes during which the Hamiltonian remains unchanged and during which the responsibility for the phenomenon of the phase transition is transferred to a redefinition of the underlying physical Hilbert space. In 1992, for example, Scholtz et al [10] introduced a sophisticated non-Hermitian boson-field generalization of the so called Lipkin-Meshkov-Glick model. These authors demonstrated (cf. Figure Nr. 1 in loc. cit.) that the system exhibits a phase transition which is not caused by a modification of the operators of the observables themselves.

In the real world of experimental physics the passage of a given quantum system through its phase transition instant $t_{0}$ is usually assumed to proceed very slowly, in an adiabatic dynamical regime. Unfortunately, the authors of Ref. [44] demonstrated that in the non-Hermitian cases such an approximation strategy need not be applicable in the quasi-Hermitian quantum mechanics in general. In the light of the relevant review papers (cf., e.g., [15]), the transition from the well known Hermitian formalism of textbooks to the slightly counterintuitive quasi-Hermitian picture of dynamics may also lead to several other theoretical as well as purely mathematical consequences. On the theoretical side one must emphasize that after the passage through the interface the observability status of the energies themselves remained, by the construction of our illustrative model, unchanged. 
Formally, this means that in place of the pre-passage Hermitian lower-case matrix (3) living, by definition, in the Hermitian dynamical regime with $(c, t) \in \mathcal{D}_{\text {(conventional), }}$, i.e., with $c \leq t^{2}$, the role of the energies was changed and played by the (strictly real) eigenvalues of the new, non-Hermitian matrix (9).

The passage from model (4) to its phenomenologically acceptable continuation (9) was postulated smooth. After one leaves the safe textbook half-plane with $c \leq 0$ and after one moves to the small and positive $c$ 's, one does not observe any qualitative changes in the time-dependence of the energy levels at the instants of transition $t= \pm \sqrt{c}$. Incidentally, a similar smoothness characterizes the phase transition occurring in the Lipkin-MeshkovGlick-type model of Ref. [10] (cf. Figure Nr. 2 in loc. cit.). In our present model, this smoothness (i.e., the phase transition of the second kind [4]) is illustrated by Fig. 4] where the smooth spectral shape does not offer any indication that in the interval of times $t \in(-\sqrt{c}, \sqrt{c})$ (indicated by the two thin markers below the curves) the Hermitian matrix (3) gets replaced by its non-Hermitian continuation (91).

\section{Conclusions}

From the historical perspective it was fortunate that during the birth of quantum mechanics people did not pay too much attention to the elementary hydrogen-type quantum systems in which the electrons (= fermions) would be replaced by pions (i.e., bosons). This would almost certainly slow down the early stages of development. The experiments would be found to disagree with the theory and the theory would suffer from the emergence of multiple theoretical challenges including, first of al, the manifest non-Hermiticity of the underlying relativistic Klein-Gordon Hamiltonian (cf., e.g., pp. pp. $357-360$ in [3] for more details). Some of these conceptual questions remained, for a long time, unanswered (cf., e.g., the related remark on p. 349 in [3]).

The much-delayed consequent resolution of the problem was only published, cca 15 years ago, in Ref. [18]. An acceptable probabilistic interpretation of the pionic-atomlike quantum mechanics was based there, in essence, on the application of the concept of the quasi-Hermiticity. Unfortunately, the solution of the Klein-Gordon puzzle was still incomplete, based on the very strong assumption that the system in question is static (cf., e.g., Theorem 2 in Ref. [12]). In other words, for the pionic atoms the Klein-Gordon Hamiltonians were only shown to describe the critical quantum phenomena (like, e.g., the complexification of the energies) in an adiabatic approximation.

The approximation-free $3 \mathrm{HS}$ formalism able to provide a complete description of the processes of quantum degeneracies has only been formulated very recently (see, e.g., 
Ref. [15], with further references listed therein). In our present paper we imagined that it would be desirable to apply such a formalism to the evolution of the systems which happen to pass from the Hermitian to quasi-Hermitian dynamical regime. Via an elementary illustrative example we explained the key ideas of the approach and we demonstrated that the detailed description of an elementary toy model is able to throw light on multiple conceptual questions. Among them, we made it clear that

- the analysis restricted to the mere description of the energy levels need not provide any hint that the system is going to pass through a quantum phase transition;

- the traditional textbook versions of quantum theory based on the work with the fixed physical Hilbert space and with the operators of observables which are self-adjoint in this space admit extensive generalizations;

- one of these generalizations has been developed and discussed here via an elementary benchmark model in which the dynamical input knowledge has the form of a given non-Hermitian and time-dependent energy operator $H(c, t)$ with real spectrum.

At the end, the study led to the rather optimistic conclusion that the $3 \mathrm{HS}$ formalism is able to provide a consistent and mathematically correct representation of physical reality in which the description of the passage of a quantum system through its Hermitianquasi-Hermitian interface exhibits a number of close analogies with its simpler, adiabaticapproximation predecessors.

\section{Acknowledgments}

The work was supported by the GAČR Grant Nr. 16-22945S. 


\section{References}

[1] A. Messiah, Quantum Mechanics I. Amsterdam, North Holland, 1961.

[2] S. Flügge, Practical Quantum Mechanics II. Springer, Berlin, 1971.

[3] F. Constantinescu and E. Magyari, Problems in Quantum Mechanics. Pergamon, Oxford, 1971.

[4] D. I. Borisov, Acta Polytech. 54, 93 (2014);

D. I. Borisov, F. Růžička and M. Znojil, Int. J. Theor. Phys. 54, 4293 (2015).

[5] M. Znojil, Ann. Phys. (NY) 336, 98 (2013);

D. I. Borisov and M. Znojil, in Non-Hermitian Hamiltonians in Quantum Physics, F. Bagarello, R. Passante and C. Trapani, eds. Springer, Berlin, 2016, pp. 201 - 217.

[6] H. Eleuch and I. Rotter, Phys. Rev. A 95, 022117 (2017).

[7] N. Moiseyev, Non-Hermitian Quantum Mechanics. Cambridge University Press, Cambridge, 2011.

[8] C. M. Bender and S. Boettcher, Phys. Rev. Lett. 80, 5243 (1998).

[9] H. F. Jones, Phys. Rev. D 78, 065032 (2008).

[10] F. G. Scholtz, H. B. Geyer and F. J. W. Hahne, Ann. Phys. (NY) 213, 74 (1992).

[11] C. M. Bender, Rep. Prog. Phys. 70, 947 (2007).

[12] A. Mostafazadeh, Int. J. Geom. Meth. Mod. Phys. 7, 1191 (2010).

[13] M. Znojil, Phys. Rev. D 78, 085003 (2008).

[14] M. Znojil, SIGMA 5, 001 (2009) (e-print overlay: arXiv:0901.0700).

[15] M. Znojil, Ann. Phys. (NY) 385, 162 (2017).

[16] F. Bagarello, J.-P. Gazeau, F. H. Szafraniec, and M. Znojil, Non-Selfadjoint Operators in Quantum Physics: Mathematical Aspects. Wiley, Hoboken, 2015.

[17] P. Dorey, C. Dunning and R. Tateo, J. Phys. A: Math. Theor. 40, R205 (2007). 
[18] A. Mostafazadeh, Class. Quant. Grav. 20, 155 (2003);

A. Mostafazadeh, Ann. Phys. (N.Y.) 309, 1 (2004);

M. Znojil, J. Phys. A: Math. Gen. 37, 9557 (2004).

[19] M. Znojil, J. Phys. A: Math. Theor. 45, 444036 (2012);

G. Lévai, F. Růžička and M. Znojil Int. J. Theor. Phys. 53, 2875 (2014).

[20] Y. N. Joglekar, D. Scott, M. Babbey and A. Saxena, Phys. Rev. A 82, 030103 (2010);

Y. N. Joglekar, C. Thompson and G. Vemuri, Phys. Rev. A 83, 063817 (2011);

F. Růžička, Int. J. Theor. Phys. 54, 4154 (2015);

T. E. Lee and Y. N. Joglekar, Phys. Rev. A 92, 042103 (2015);

A. K. Harter, T. E. Lee and Y. N. Joglekar, Phys. Rev. A 93, 062101 (2016).

[21] R. El-Ganainy, K. G. Makris, D. N. Christodoulides and Z. H. Musslimani, Opt. Lett. 32,2632 (2007);

K. G. Makris, R. El-Ganainy, D. N. Christodoulides and Z. H. Musslimani, Phys. Rev. Lett. 100, 103904 (2008);

A. Guo, G. J. Salamo, D. Duchesne, et al, Phys. Rev. Lett. 103, 093902 (2009);

Ch. E. Rüter, K. G. Makris, R. El-Ganainy et al., Nature Physics 6, 192 (2010);

E. M. Graefe and H. F. Jones, Phys. Rev. A 84, 013818 (2011);

A. Mostafazadeh and S. Rostamzadeh, Phys. Rev. A 86, 022103 (2012);

A. Mostafazadeh, Phys. Rev. A 87, 012103 (2013);

M. Znojil, Symmetry 8, 52 (2016);

R. El-Ganainy, K. G. Makris, M. Khajavikhan et al., Nat. Phys. 14, 11 (2018).

[22] H. F. Jones, Phys. Rev. D 76, 125003 (2007).

[23] M. Znojil, Phys. Lett. A 259, 220 (1999);

V. Jakubský, M. Znojil, E. A. Luis et al, Phys. Lett A 334, 154 (2005).

[24] D. Krejčiřík, H. Bíla and M. Znojil, J. Phys. A: Math. Gen. 39, 10143 (2006);

B. Bagchi, S. Mallik, H. Bíla et al, Int. J. Mod. PHys. A 21, 2173 (2006).

[25] M. Znojil and V. Jakubský, J. Phys. A: Math. Gen. 38, 5041 (2005);

V. Lotoreichik and P. Siegl, Proc. AMS 145, 1231 (2017). 
[26] M. Znojil, e-print arXiv: 1710.03470v2.

[27] M. H. Stone, Ann. Math. 33, 643 (1932).

[28] J. Dieudonné, in Proc. Int. Symp. Lin. Spaces. Oxford, Pergamon, 1961, p. 115.

[29] D. Krejčiř́ík and P. Siegl, in Ref. [16], pp. 241 - 292.

[30] M. Znojil, in Ref. [16], pp. 7 - 58.

[31] C. Figueira de Morisson Faria and A. Fring, Laser Phys. 17, 424 (2007);

M. Znojil, Int. J. Theor. Phys. 52, 2038 (2013).

[32] J.-B. Gong and Q.-H. Wang, Phys. Rev. A 82, 012103 (2010);

J.-B. Gong and Q.-H. Wang, J. Phys. A: Math. Theor. 46, 485302 (2013).

[33] M. Maamache, Phys. Rev. A 92, 032106 (2015);

M. Maamache, Acta Polytech. 57, 424 (2017).

[34] A. Fring and M. H. Y. Moussa, Phys. Rev. A 93, 042114 (2016);

A. Fring and M. H. Y. Moussa, Phys. Rev. A 94, 042128 (2016).

[35] F. J. Dyson, Phys. Rev. 102, 1217 (1956).

[36] M. Znojil, Phys. Lett. A 379, 2013 (2015).

[37] H. Bíla, Non-Hermitian Operators in Quantum Physics. Charles University, Prague, 2008 (PhD thesis);

H. Bíla, e-print arXiv: 0902.0474.

[38] E. Schrödinger, Ann. d. Phys. 81, 109 (1926).

[39] W. Heisenberg, Z. Phys. 33, 879 (1925).

[40] D. F. Styer, M. S. Balkin, K. M. Becker et al, Am. J. Phys. 70, 288 (2002).

[41] Y.-G. Miao and Zh.-M. Xu, Phys. Lett. A 380, 1805 (2016).

[42] A. Fring and T. Frith, Phys. Rev. A 95, 010102(R) (2017).

[43] T. Kato, Perturbation theory for linear operators. Springer, Berlin, 1966.

[44] T. J. Milburn, J. Doppler, C. A. Holmes, S. Portolan, S. Rotter and P. Rabl, Phys. Rev. A 92, 052124 (2015). 